Aus dem Sanatorium für innere und Nervenkrankheiten Schloß Hornegg a. N.

\section{Zur Klinik postdiphtherischer Pseudotabes (Liquorbefunde bei postdiphtherischer Lähmung.')}

Von Dr. L. Roemheld.

Bei der großen Aehnlichkeit des klinischen Bildes der Tabes dorsalis einerseits und der schweren Formen postdiphtherischer Lähmungen anderseits, die man gelegentlich analog der alkoholischen Polyneuritis als $\mathrm{P} s$ e u d o ta b e s bezeichnet findet, mußte es von Interesse sein zu untersuchen, ob auch das Verhalten des Liquor cerebrospinalis eine gewisse Uebereinstimmung zeige. Soviel mir bekannt, sind über diesen Punkt noch keine Untersuchungen angestellt worden, wie überhaupt die Literatur über die Beschaffenheit der Zerebrospinalflüssigkeit bei akuten Infektionskrankheiten noch recht klein ist. Und gerade über Liquorbefunde bei Diphtherie liegen bis dahin noch keine Beobachtungen vor. Es leuchtet ein, dab solche Untersuchungen nicht nur ein kasuistisches Interesse beanspruchen, sondern dab ihnen eine gewisse prinzipielle Bedeutung zukommt, da sie zur Klärung mancher noch strittiger Fragen herangezogen werden können.

Heute unterliegt trotz zahlreicher anatomischer und experimenteller Untersuchungen die Frage nach dem Sitz postdiphtherischer Lähmungen immer noch der Kontroverse. Klinisch, unter Zuhilfenahme von Lumbalpunktionen ist die Frage bis dahin noch nicht in Angriff genommen worden. Einige neuere Autoren, so besonders Spieler (1), kommen zu der Annahme, daB in den entsprechenden Frühstadien der Lähmungen peripherische Nervenveränderungen nachweisbar sind. Anderseits sind aber bei länger dauernden postdiphtherischen Lähmungen anatomische Störungen in den Zentralorganen selbst geradezu ein klinisches Postulat. Denn nur durch zentrale Degeneration kann man die Ausdehnung der Lähmungen, das Verschwinden der Sehnenreflexe, das Ergriffensein der verschiedensten Nervengebiete erklären. Baginsky (2) spricht sich deshalb in seiner Monographie über Diphtherie auch dahin aus, daß es sich wohl im Beginn um eine Neuritis ascendens toxica handle, daß diese sich aber dann im Gebiet des Neurons weiter fortpflanzt und schließlich zu einer Läsion der zentralen Apparate bis zu einer Vernichtung derselben führen kann. Aeltere experimentelle Untersuchungen von $M$ u rawjeff (3) scheinen diese Annahme zu stützen, denn sie haben dargetan, daß beim Meerschweinchen unter Einwirkung des Diphtheriegiftes die anterolaterale Zellgruppe der Vorderhörner am leichtesten geschädigt wird, daB aber gleichzeitig auch die peripherischen Nerven durch fettige Degeneration lädiert werden.

Auch neuere experimentelle Forschungen von Crocq, Foulerton, Thomson, Ransom haben neuritische Prozesse, daneben aber auch Veränderungen in den Wurzeln, den Meningen und im Rückenmark selbst nachgewiesen, und Babon neix und Sano (4) haben, letzterer in dem Oppenheimschen Laboratorium, starke Entartungsprozesse an den Rückenmarkswurzeln und im Rückenmark selbst aufgedeckt, während wieder in anderen Fällen, wie Kohts und $\mathrm{Hasche}$ (4) beschreiben, der Befund ein völlig negativer war.

Bei Typhus, Influenza, Keuchhusten, Parotitis epidemica und Pneumonie hat man Veränderungen des Liquors gefunden. Zu welchem Zeitpunkt der Erkrankung diese Veränderungen auftreten, $o b$ sie rein toxisch bedingt sind, oder ob sie durch septisch-entzündliche Prozesse an den Zentralorganen und an den Meningen verursacht werden, wissen wir nicht. Wir sind darüber bis jetzt ebenso im unklaren wie über die Frage, auf welche Weise bei frischer Lues und bei metasyphilitischen Prozessen die Lymphozytose

1) Nach einem Vortrag, gehalten auf der II. Jahresversammlung der Gesellschaft Deutscher Nervenärzte am 3. Oktober 1908 in Heidelberg. und die Vermehrung des Eiweibgehaltes im Liquor zu. stande kommt.

Während die einen, so besonders Nissl (5) und Merzbacher $(6,7)$, annehmen, daß ïberhaupt die Infeltion als eine Noxe anzusehen sei, die unabhängig von den klinischen Symptomen eine zerebrospinale Pleozytose erzeuge durch unbekannte Alteration des Mechanismus, durch dessen Tätigkeit die Lymphozyten in die Zerebrospinalflüssigkeit gelangen, behaupten andere, so vor allem die Franzosen und in Deutschland neuerdings nachdrücklich $F$ is cher (8) in Prag, daß der Zellvermehrung stets eine hypothetische meningitische Reizung bzw. Entzündung zugrunde liegt.

Möglicherweise können später auch zur Lösung dieser Frage Beobachtungen bei akuten Infektionskrankheiten, speziell bei Diphtherie und postdiphtherischen Lähmungen herangezogen werden. Meine Mitteilungen über Liquorbefunde bei postdiphtherischer Lähmung regen vielleicht dazu an, bei einem größeren klinischen Material in geeigneten Fällen von Diphtherie Lumbalpunktionen vorzunehmen, um so zu eruieren: ob Liquorveränderungen konstant auftreten, ferner ob sie sich erst im Stadium der Lähmung oder schon vorher einstellen, und durch pathologisch-anatomische Untersuchungen der $\mathrm{Me}$ ningen von Patienten, die an Diphtherie gestorben sind, festzustellen, ob meningeale Veränderungen Ursache der Lymphozytose und der Eiweißvermehrung sind oder nicht.

Mein Patient, ein 30 jähriger Kaufmann, aus gesunder Familie stammend und selbst früher stets gesund, speziell nicht luetisch infiziert, erkrankte Ende November 1907 an schwerer Diphtherie und erhielt von dem Hausarzt am ersten Krankheitstag Behrings Serum in einer Stärke von 2000 I.-E. injiziert. Rasche Entfieberung. Nach 14 Tagen ging der Patient wieder gegen den ärztlichen Rat ins Geschäft. Um diesen Zeitpunkt stellte sich eine Sehstörung ein, die Sprache wurde nasal, der Hausarzt konstatierte eine Gaumens egellähmung, der zugezogene Augenarzt eine Akkommodationslähmung auf beiden Augen ohne Pupillenstörung. Mitte Januar 1908 fiel der Ungebung zuerst der unsichere Gang des Patienten auf, der einen so hohen Grad annahm, daß der Kranke kaum mehr allein gehen konnte. Am 30. Januar Aufnahme in meine Anstalt.

Wir fanden bei dem mittelkräftig gebauten Herrn an den inneren Organen nichts Abnormes. Speziell ergab die Untersuchung des Herzens normalen Befund, der Blutdruck betrug $100 \mathrm{~mm} \mathrm{Hg}$ nach Riva-Rocci, die Uvula stand gerade, hob sich beim Phonieren nicht sehr hoch, der rechte Gaumenbogen war mehr beweglich als der linke, die Sprache noch deutlich nasal. Augenbefund normal; gesteigerte mechanische Erregbarkeit im Facialisgebiet. An den oberen Extremitäten war die motorische Kraft herabgesetzt, a usges prochene A taxie, ganz unleserliche Schrift, Sensibilität für alle Qualitäten normal, Reflexe schwach, Nervenstämme auf Druck nur wenig empfindlich. Bauch- und Cremasterreflex wenig lebhaft. An den unteren Extremitäten wardie Ataxie noch größer, sodaß der Patient nur mit Mühe gehen konnte. Es bestand starke Hy potonie, Patellar-und Achillesreflex fehlten beiderseits; desgleichen Babinski; BechterewMendel dorsal. Außer leichter Hyperaesthesie keine objektiven Sensibilitätsstörungen, dagegen subjektiv Ameisenlaufen. Auch an den Fußsohlen normale Sensibilität, keine Störung der tiefen Sensibilität. Romberg positiv. Patient konnte nicht auf einem Bein stehen. Die elektrische Untersuchung ergab geringe Herabsetzung der elektrischen Erregbarkeit, keine qualitative Veränderung. Blasen-, Mastdarm- und Geschlechtsfunktion normal.

Am 11. Februar wurde die erste Lumbalpunktion vorgenommen. Der Liquor entleerte sich unter mäßigem Druck. Es wurden $6 \mathrm{ccm}$ entnommen, die zur Kontrolle gleichzeitig bei uns und in der psychiatrischen Klinik in Heidelberg ${ }^{1}$ ) vorgenommene Untersuchung ergab: 1. Mäßige Lymphozytose nach Nisslscher Bezeichnung. ${ }^{2}$ ) Keine Häufchenbildung, keine Leukozytose. Zählung in der Rosenthalschen Kammer ergibt im ganzen 20, also im $\mathrm{mm}^{8}$ 6-7 Lymphozyten gegenüber $0,5-2$ Zellen im $\mathrm{mm}^{2}$ unter normalen Verhältnissen. 2. Starke Eiweißvermehrung, über sieben Teilstriche der Nisslschen Skala; 3. die Nonnesche Phase I-Reaktion stark positiv.

Im v. Dungerschen Laboratorium des Heidelberger Krébsinstituts wurde ferner festgestellt, da $\beta 4$. die Verstärkung der hemmenden Wirkung des Lecithins im haemolytischen System ausblieb.

Der Patient wurde inzwischen bei uns mit Strychnin-Injektionen, elektrischen Bädern und leichter Gymnastik behandelt. Der Zustand besserte sich sichtlich. Am 10. März zweite Lumbalpunktion.

1) Herrn Dozenten Dr. Ranke bin ich für die Kontrolluntersuchuugen zu besonderem Dank verpflichtet. - 2) D. h. im Immersionsgesichtsfeld mit Okular 1 5-7 Lymphzoyten im Gesichtsfeld, wenn man aus dem Zentrifugat 3-4 Tropfen macht.: 
Untersuchung, wie bei der ersten Punktion vorgenommen, ergibt:

1. Lymphozyten nach dem Befund der psychiatrischen Klinik in Heidelberg vielleicht noch eine Spur vermehrt;

2. Eiweißgehalt vier Teilstriche der Nisslschen Skala;

3. Phase I-Reaktion zeigt Opaleszenz;

4. die v. Dungernsche Probe ist negativ;

5. Diptherietoxin und -antitoxin fehlen im Liquor (untersucht im Krebsinstitut).

Nach diesem Befund war ein völliger Parallelismus zwischen dem Zurackgehen der klinischen Erscheinungen und der Veränderung der Liquorbestandteile zu konstatieren.

Am 8. April finde ich in meinem Journal notiert, daß die $\mathrm{Pa}-$ tellarreflexe zum erstenmal auslosbar waren. Am 14. April wurde der Patient anf seine Bitte mit folgendem Abgangsbefund entlassen:

Gewichtszunahme hier 9 Pfund. Der Patient kann ohne Ermüdung zwei Stunden weit gehen. Uvula vollig normal, kein Verschlucken mehr. An den oberen Extremitäten keine Ataxie, Schrift normal. Der Patient ist a uf den Beinen völlig sicher, geht mit geschlossenen Augen die Treppe hinauf und herunter. Romberg negativ. Motorische Kraft normal, desgleichen die Sensibilität. Die Patellarreflexe sind deutlich vorhanden, aber schwach, der linke ist deutlicher als der rechte, der nur mit Jen drassik anslossbar ist. Achillesreflex fehlt noch beiderseits.

Mit Einwilligung dess Patienten wurde am Tage vor der Abreise, am 13. April, eine dritte I umbalpunktion vorgenommen, deren Ergebnis folgendes war:

1. Lymphozy ten vielleicht noch ein Minimum vermehrt, wahrscheinlich normal an Zahl; 2. Eiweißgehalt: 3 Teilstriche der Nisslschen Skala; 3. Phase I-Reaktion ergibt minimale Opaleszenz. Das Resultat war also, daß nur noch der Eiweißgehalt dentlich pathologisch war, der nach Nissl normal bis zu zwei Teilstrecken betragen darf.

\begin{tabular}{|c|c|c|c|c|c|c|}
\hline & Datum & Zeligehalt & $\begin{array}{l}\text { Eiweik- } \\
\text { gehalt }\end{array}$ & $\begin{array}{l}\text { Phase I- } \\
\text { Reaktion }\end{array}$ & $\begin{array}{l}\text { Lecithin- } \\
\text { Reaktion }\end{array}$ & $\underset{\text { toxin }}{\text { Diphtherie- }}$ \\
\hline 1. & 11. Febr. 08 & $\begin{array}{c}\text { MäBige } \\
\text { Lymphozytose, } \\
6-7 \text { Zeilen } \\
\text { im } \mathrm{mm}^{3}\end{array}$ & $\begin{array}{l}\text { 7 Teilstriche } \\
\text { nach Niss }\end{array}$ & $\dagger+$ & fehlt & - \\
\hline 2. & 10. März 08 & $\begin{array}{c}\text { Minimale } \\
\text { Vermehrung }\end{array}$ & 4 Teilstriche & Opaleszenz & fehlt & fehlt \\
\hline 3. & 13. April & Normal & 3 Teilstriche & $\begin{array}{c}\text { Minimale } \\
\text { Opaleszenz }\end{array}$ & - & - \\
\hline
\end{tabular}

Rekapitulieren wir nochmals in Kürze, so ergibt unsere Beobachtung, daß sich bei einem erwachsenen Patienten im Anschluß an eine schwere, mit Behrings Serum behandelte Diphtherie im Laufe von $2-3$ Monaten $A \mathrm{kkommodations-}$ lähmung, Gaumensegellähmung und im übrigen das Bild einer Pseudotabes entwickelt hat, als deren Grundlage wir nach der seitherigen Auffassung eine peripherische Neuritis anzusprechen hätten. Die Druckempfindlichkeit der Nervenstämme, das Fehlen der Reflexe, die Herabsetzung der elektrischen Erregbarkeit, die allerdings fast nur subjektiven Sensibilitätsstörungen lassen keine andere Deutung zu. Daß objektiv die Sensibilitätsstörungen so wenig ausgeprägt waren, deckt sich mit ähnlichen Beobachtungen von Baginsky, Erb, (9) Henoch (10). Die ungewöhnlich starke Ataxie ergänzte das Bild der Pseudotabes.

Ueber die Differentialdiagn ose unseres Falles brauchte ich wohl kein Wort zu verlieren. Dafür, daß es sich nicht um einen metasyphilitischen Prozeb gehandelt hat, spricht der Verlauf und die Anamnese, in der Lues fehlt. Auch mügte man nach dem heutigen Stand unserer Kenntnisse schon aus der schrittweise verfolgten Rückkehr der Lymphozytose zur Norm schließen, daß unser Patient niemals eine spezifische Infektion erlitten hat.

Bei dem geschilderten klinischen Bild fand sich nun eine starke, bis jetzt noch nicht beobachtete pathologische Veränderung der Zerebrospinalflüssigkeit, die sich besonders in Vermehrung des Eiweißgehaltes, weniger in Vermehrung der zelligen Elemente äußerte und Hand in Hand mit der klinischen Besserung einen Rückgang zeigte. Die Eiweibvermehrung war anfangs so bedeutend, wie man sie nur bei Paralyse oder Meningitis zu finden gewohnt ist, während die Lymphozytose schon bei der ersten Untersuchung sich in sehr mäßigen Grenzen hielt.

Ich unterlasse es, auf eine Reihe von Fragen, die sich einem bei Betrachtung der Krankengeschichte unwillkürlich aufdrängen, einzugehen. Von Interesse wäre es zu untersuchen, $\mathrm{ob}$ es überhaupt möglich ist, daß eine so hochgradige Ataxie, wie wir sie bei unserem Patienten trotz normaler Fußsohlen- und Tiefensensibilität fanden, nur peripherische Ursachen hat. Auch auf die Tatsache, dab die Nonnesche Phase I-Reaktion in unserem Falle positiv war, sei kurz hingewiesen. Unsere Beobachtung scheint mir aber, abgesehen von dem kasuistischen Interesse, als erste Mitteilung über den Lumbalpunktionsbefund bei postdiphtherischer Lähmung nach den beiden oben angedeuteten Richtungen hin verwertbar zu sein. Einmal stellt sie einen klinischen Beitrag zur Lösung der Frage nach dem Sitz postdiphtherischer Lähmungen dar, und zweitens lassen sich vielleicht aus solchen Beobachtungen bei akuten Infektionskrankheiten Rückschlüsse auf die Entstehung der Lymphozytose und der Eiweißvermehrung bei metasyphilitischen Erkrankungen ziehen.

Alle Liquoruntersuchungen bei peripherischer Neuritis (ausführliche Literaturangaben bringt Merzbacher) von Babinski, Nageotte, Sicard, Laignel-Lavastine, Nissl sind bis jetzt negativ ausgefallen. Nur in einem Falle von Bleilähmung hat Sicard (11) Lymphozytose konstatieren kơnnen. Bei Bleiintoxikation haben ubrigens neuerdings Mosny und Malloizel (12) immer Lymphozytose gefunden, die sie auf meningitische Reizung beziehen. Auch uber Eiweißvermehrung im Liquor bei gewőhnlicher Neuritis ist in der Literatur nichts bekannt.

Handelte es sich also bei postdiphtherischen Lähmungen einfach um peripherische Neuritis, etwa wie die alkoholische Neuritis, so wäre nicht verständlich, warum es gerade bei dieser Form der peripherischen Nenritis zu solchen Veränderungen des Liquors kommt. Wir werden deshalb, so weit eine einzelne klinische Beobachtung einen Schluß zuläßt, annehmen dürfen, dab bei postdiphtherischen Lähmungen, wenigstens wenn sie schon längere Zeit bestehen, keine einfache peripherische Erkrankung mehr vorliegt. Die Liquorveränderung weist vielmehr ähnlich wie bei metasyphilitischen Erkrankungen auf zentrale Prozesse hin.

Vielleicht darf man die Befunde bei chronischer Bleiintoxikation und bei Herpes zoster hierzu in eine gewisse Parallele setzen.

In unserem Fall war im Liquor bei der zweiten Lumbalpunktion, die $3^{1} / 2$ Monate nach Beginn der Erkrankung vorgenommen wurde, kein Diphtherietoxin mehr nachweisbar. Möglicher'weise hätte es zu einem früheren Zeitpunkt gefunden werden können. Die Hochgradigkeit der Liquorveränderung, namentlich die beträchtliche Eiweißvermehrung, zu einer Zeit, wo Diphtherietoxin im Liquor fehlte, macht es zum mindesten unwahrscheinlich, daß es sich hier bei den zentralen Prozessen, die wir nach dem Vorhergesagten annehmen müssen, nur um toxisch bedingte Störungen der Regulationsvorrichtungen gehandelt hat, welche normalerweise die konstante Zusammensetzung des Liquors gewährleisten. Man muß in einem solchen Fall wohl eher daran denken, daß die nervösen Zentralorgane und ihre Häute, wie vielleicht auch bei den anderen akuten Infektionskrankheiten, durch septisch entzündliche Prozesse in Mitleidenschaft gezogen worden sind, sodal es zu zentralen anatomischen Veränderungen gekommen ist. Ein absolut sicherer Beweis für die Annahme einer meningitischen Genese der Liquorveränderung dürfte allerdings klinisch nicht möglich, sondern nur pathologisch-anatomisch zu erbringen sein. $\mathrm{Zu}$ welchem Zeitpunkt die Liquorveränderung speziell bei der Diphtherie einsetzt, darüber sagt unsere Beobachtung nichts aus, sodaß weiter auch die Frage offen bleiben muß, ob die Alteration des Liquors Folge einer Diphtheriesepsis im Beginn der Erkrankung ist, oder ob sie erst im Stadium der postdiphtherischen Lähmung durch as. zendierende Entzündung zustande kommt. Vielleicht hätte man ja bei einer früher vorgenommenen Punktion in unserem Falle noch bedeutendere Veränderungen des Liquors gefunden.

Gegenüber den metasyphilitischen Prozessen zeigt unser Fall trotz der scheinbaren oberflächlichen Aehnlichkeit des klinischen Bildes mit Tabes dorsalis einen großen Unterschied. Bei den metasyphilitischen Prozessen bleibt der pathologische Liquorbefund im großen und ganzen konstant, wenn auch 
Schwankungen vorkommen; bei postdiphtherischer Lähmung nimmt die Zerebrospinalflüssigkeit parallel mit der klinischen Besserung wieder normale Beschaffenheit an, obwohl die Veränderungen, namentlich was den Eiweißgehalt anlangt, auf der Höhe der Erkrankung bedeutender sind als bei Tabes. Bei den postdiphtherischen Lähmungen haben wir es eben weitaus in den meisten Fällen mit reparablen, vorübergehenden Störungen und anatomischen Veränderungen zu tun, die zwar, wie wir aus unserer Beobachtung schließen dürfen, auch zum Teil zentralen Sitz haben, während die metasyphilitischen Prozesse eine dauernde Schädigung des Nervensystems bedingen.

Als eine wertvolle Ergänzung unserer Beobachtung sei noch kurz folgende Krankengeschichte, die ich Herrn Prof. Niss l verdanke, mitgeteilt. Es handelte sich um einen Fall von Imbezillität, die sich im AnschluBan eineschwere Diphtherie mit postdiphtherischer Lähmung entwickelt hatte und bei welcher ebenfalls eine pathologische Liquorveränderung konstatiert wurde.

A. N., 18 Jahre alt, stammt aus gesunder Familie. Eltern nicht verwandt. Patient war als Kind bis zum vierten Jahr gut entwickelt und anscheinend normal veranlagt. Mit vier Jahren schwere Diphtherie, danach Rekonvaleszenz etwa \% Jahr lang. Dann Schwäche in den Beinen. Die Eltern glaubten erst, das Kind sei faul, wolle nicht gehen, prügelten es. Dann aber stellte sich eine schlaffe Lähmung der Beine ein ohne Schmerzen. Die Störung bildete sich langsam zurück, doch machte sich nach der Diphtherie ein Stillstand der geistigen Entwicklung bemerkbar. Der Patient blieb in der Schule weit hinter den Leistungen seiner Mitschüler zurïck. Er besuchte die Volksschule sieben Jahre lang, hatte aber stets nur schlechte Leistungen auf $\_$uweisen. Auch in der Lehre bei einem Tapezierer waren die Leistungen völlig ungenügend. $\mathrm{Er}$ war in seinem Benehmen kindisch, spielte gerne mit Kindern, war meist verträglich, bisweilen auch aufgeregt. Dabei absolut unselbständig und vollkommen interesselos.

Veranlassung zur Einlieferung in die Klinik war die Belästigung eines Mädchens auf der Straße durch den Patienten.

Aufnahme am 28. Februar 1908.

Orientiert; meint, er sei im Krankenhaus, sei wegen Katarrhs hier zur Erholung. Gang trippelnd. Ist sehr redselig. Unzählige Bitten, sehr um sich besorgt. Unruhige Bewegungen. Er ist besonnen, gibt genaue Auskunft: in der Schule sei er schlecht gewesen, im Beruf müsse er noch lernen. Als kleines Kind habe er einmal nicht gehen können, es sei aber von selbst wieder besser geworden. Eine äußere Ursache habe die Krankheit nicht gebabt. Intelligenz sehr schwach, Rechnen schlecht, sehr langsam; Geographiekenntnisse mäßig. Körperlich: kleiner Schädel, etwas asymmetrisches Gesicht. Klagt über häufiges Kopfweh. Reflexe links = rechts. Pupillen reagieren, Sensibilität intakt. Herz normal.

24. März. Neurologische Untersuchung: Sehnenreflexe wenig lebhaft, doch nicht unternormal. Bein-, Armmuskulatur schlecht entwickelt, nicht atrophisch. Infantiler Habitus der Muskulatur. Hautreflexe vorhanden, beiderseits gleich. Kyphoskoliose, rachitischer Thorax. Keine Zeichen von Rachitis an den Epiphysun. Eigentümlicher Gang, nach der Mittellinie konvergierende Finger. Nägel nicht rissig. Sehr degeneriertes Gebiß, Degenerationsspalte beim Aufeinanderbeißen der Schneidezähne, vorstehende Eckzähne, verbildete Ohren, Haare auf der Nasenwurzel, keine Sensibilitätsstörungen, Augenhintergrund normal, Astigmatismus beiderseits.

A pril 1908. Sitzt auf der Abteilung herum, beschäftigt sich kaum, höcbstens mit dem Betrachten von Bildern, von denen er nichts versteht. Gelegentlich Wünsche wegen des Essens. Geht mit Mutter und Schwester hier und da in die Stadt spazieren, freut sich darauf besonders deshalb, weil er dann seine eigene Mütze aufsetzen darf.

Kopfschmerz und Schwindel wird in der Klinik nicht beobachtet, dagegen erzahlt er gelegentlich davon, $\mathrm{daB}$ er als Kind viel an Kopfschmerzen gelitten und deshalb nur wenig gelernt habe.

Lumbalpunktionen wurden vorgenommen am 16. März 1908 und am 28. April 1908. Es fand sich das erstemal: Starke Lymphozytenvermehrung, zahlreiche Häufchen, 4,2 Teilstriche Eiweißgehalt; bei der zweiten Punktion: leichte Blutbeimengung, deutliche Vermehrung der Lymphozyten, einzelne Häufchen, 5 Teilstriche Eiweißgehalt.

Wenn diese zweite Beobachtung auch nicht so beweisend ist wie die erste, so geht doch jedenfalls mit Sicherheit aus der Krankengeschichte hervor, dab der Patient im jugendlichen Alter eine schwere Diphtherie mit folgender Lähmung überstanden hat. Da mit Bestimmtheit angegeben wird, daß er als Kind vor Eintritt der Diphtherie geistig normal gewesen sei, und da von einer anderen Krankheit, die zur Imbezillität geführt haben könnte (Lues, Meningitis, Encephalitis, Poliomyelitis etc.), nichts zu eruieren ist, darf man mit großer Wahrscheinlichkeit annehmen, daB im AnschluB an die Diphtherie eine organische Veränderung der Zentralorgane stattgefunden hat, als deren klinische Residuen wir jetzt noch, nach 14 Jahren, den vermutlich fortschreitenden Schwachsinn und die Liquorveränderung konstatieren können, während von der Lähmung der Beine nichts mehr nachzuweisen ist. Welcher Art die Veränderung des Zentralorgans war, vermögen wir nicht zu sagen. Herderscheinungen fehlen jetzt; sonach liegt es nahe, an diffuse organische Veränderungen der Hirnrinde und an chronisch-meningitische Zustände zu denken, welche wir wohl als Ueberreste der durch die infektiöse Krankheitsursache bewirkten Ernährungsstörungen im Zentralnervensystem aufzufassen haben.

Der Fall würde sonach noch mehr als unsere erste Beobachtung dafür sprechen, dab die Veränderungen des Liquors bei Diphtherie nicht einfach toxisch bedingt sind, sondern auf anatomischen Läsionen der Zentralorgane und ihrer Häute beruhen.

Während aber bei unserer ersten Beobachtung, wie es wohl das Gewöhnliche ist, alle klinischen Symptome und Hand in Hand damit auch die Liquorveränderungen sich zurückgebildet haben, ist hier nach dem Verschwinden der Lähmungen ein ungewöhnlich schweres Krankheitsbild zur ückgeblieben, ein stationärer Zustand von Imbezillität, von Pleozytose und von starker Vermehrung des Eiweißgehaltes. Die anatomischen Veränderungen des Zentralorgans, die wir nach den früheren Erörterungen bei postdiphtherischen Lähmungen als sicher anzunehmen haben, haben sich in unserem zweiten Fall nur teilweise zurückgebildet, Läsionen der Hirnrinde und vielleicht der Meningen sind zurückgeblieben. So kann diese zweite Beobachtung ungezwungen in Parallele gesetzt werden mit den meningitischen Prozessen, die manche Autoren bei metasyphilitischen Erkrankungen als Ursache der Liquorveränderung annehmen.

Ergebnisse. 1. Bei postdiphtherischen Lähmungen findet man pathologische Veränderungen der Zerebrospinalflüssigkeit, die sich in starker Eiweißvermehrung, weniger in Vermehrung der zelligen Elemente äußern und die Hand in Hand mit der klinischen Besserung einen Rückgang zeigen. Nur ausnahmsweise, wenn organische Veränderungen der zentralen Apparate bestehen bleiben, scheint auch der Liquor pathologische Beschaffenheit beizubehalten.

2. Mit dem Nachweis der Liquorveränderung ist zum erstenmal der klin ische Beweis dafür erbracht, daß länger dauernde postdiphtherische Lähmungen nicht nur auf peripherischer Neuritis beruhen, sondern daß es dabei zu zentralen Veränderungen kommt.

3. Es muß weiteren klinischen und pathologisch-anatomischen Untersuchungen vorbehalten bleiben festzustellen, in welchem Stadium der Diphtherie die Liquorveränderung entsteht und ob sie rein toxisch bedingt ist oder auf meningitischer Genese beruht, welch letzteres nach unseren Beobachtungen wahrscheinlicher ist. Vielleicht lassen sich dann Analogieschlüsse zwischen den Liquorveränderungen bei akuten Infektionskrankheiten und bei metasyphilitischen Prozessen ziehen.

Literatur: l. Spieler, Arbeiten aus dam Neurologischen Institut in Wien, Festschrift 1907, Bd. 15, Teil l. - 2. B a g i n s k y, Nothnagels spezielle Pathologie und Therapie, Bd. 2, Teil 1. - 3. M u ra w j e f $\mathrm{f}$, Fortschritte der Medizin 1898, No. 3, S. 93 zitiert nach B ag i n s ky. - 4. H. O p p e n h e im, Lehrbuch der Nervenkrankheiten, Bd. 1, S. 612, Berlin 1908. - 5. N is s 1 , Die Bedeutung der Lumbalpunktion für die Psychiatrie. Zentralblatt für Nervenheilkunde und Psychiatrie 1904. - 6. Merz b a cher, Zentralblatt für Nervenheilkunde und Psychiatrie 1905/06. - 7. Merz bacher, Ergebnis der Untersuchung des Liquor cerebrospinalis. Neurologisches Zentralblatt $1 \mathrm{c04}$, No. 12. - 8. F i s c h e r, Jahrbuch für Psychiatrie und Neurologie 1906 , Bd. 27. - 9. E r b, W., Handbuch der Elektrotheraple 1886, S. 499. - 10. H e n o c h, Vorlesungen über Kinderkrankheiten, 9. Aufl. 1897, S. 750. - il. S i c a rd, le liquide céphalo-rach. Paris 1902. - 12. Mos ny und Mallo izel, la méningite saturnine. Rev. de Méd. 1907, Bd. 27, S. 505. 